Research Article

\title{
Hydrodynamic Analysis of KVLCC2 Ship Sailing near Inclined Banks
}

\author{
Weilin Luo $\mathbb{D}$, Bing Yang, and Yafeng Sun \\ School of Mechanical Engineering and Automation, Fuzhou University, Fuzhou, Fujian 350116, China \\ Correspondence should be addressed to Weilin Luo; wlluo@fzu.edu.cn
}

Received 28 November 2020; Revised 16 January 2021; Accepted 2 February 2021; Published 13 February 2021

Academic Editor: Mostafa S. Shadloo

Copyright (c) 2021 Weilin Luo et al. This is an open access article distributed under the Creative Commons Attribution License, which permits unrestricted use, distribution, and reproduction in any medium, provided the original work is properly cited.

The hydrodynamic forces of KVLCC2 ship sailing near inclined banks are calculated by using CFD based on RANS equation. Corresponding CFD uncertainty analysis is conducted according to the procedure recommended by ITTC. An unstructured grid, tetrahedral grid, is employed for discretization. To control the number of grids, global element scale factor is selected as the same as refinement ratio. In numerical simulation, straightforward and oblique navigation conditions are investigated. The variation of transverse force and yaw moment with the ship-shore distance, bank angle, water depth, and drift angle are analyzed. Both hull model and hull-propeller-rudder model are considered in numerical simulation. The simulation results show the hydrodynamic characteristics of ship sailing near inclined banks.

\section{Introduction}

Ship maneuverability is one of the most important ship hydrodynamic performances since it relates closely to the safety and economy of ship navigation. In 2002, International Maritime Organization (IMO) promulgated the Ship Manoeuvrability Standard and recommended the prediction of ship manoeuvrability at the stage of ship design [1]. Since then, the study on the prediction of ship manoeuvrability has been paid attention to increasingly. Meanwhile, advices on the modification of the Standard have also been put forward because the Standard can only be applied to the infinite waters and calm waters. Many studies have confirmed that ship manoeuvrability in restricted waters is quite different from that in infinite waters. Typical restricted waters refer to narrow and/or shallow waters. Squat and/or bank suction phenomenon might occur in such waters, which form a threat to the safety of ship navigation [2]. In order to avoid the collision between ships and channel bottoms or banks, it is of great significance to study the hydrodynamic characteristics of the ships sailing in restricted waters and to predict the ship manoeuvrability in restricted waters. In the study, the main concern is the ship manoeuvring hydrodynamics in specific restricted waters, i.e., waters near inclined banks, which are commonplace in harbors. Due to the asymmetric flow field around a ship in the vicinity of an inclined bank, the bank effect is obvious. A reliable hydrodynamic prediction can help determine how the ship can safely navigate in such a waterway.

It is known that simulation-free and simulation-based methods are available for the prediction of ship manoeuvrability [3]. Simulation-free methods come to the experiments while the simulation-based methods refer to systembased manoeuvring simulation and computational fluid dynamics- (CFD-) based manoeuvring simulation. In the experiments on ship manoeuvrability in narrow waters, a representative work was conducted by Norrbin [4] who carried out the experimental research on the hydrodynamic force on a tanker model when it moved along the vertical bank, the sloping bank, and the stepping water bottom. He analyzed the influence of the shape of the bank, the depth of water, and the ship-bank distance on the ship's transverse force and the yaw moment and obtained the regression formula from a large number of experimental data, which provides an important reference for navigators, managers, and researchers. Lataire et al. [5] carried out an extensive set of model tests to investigate bank effects induced by sloped surface-piercing as well as submerged banks. Gronarz [6] 
studied the effects of drift angle, ship-bank distance, and ship speed on near-bank navigation of ships in shallow water by towing-type tank tests and analyzed the coupling effect of transverse force and yaw moment. Compared with experiment-based simulation-free method, the main advantage of simulation-based approach to predicting ship manoeuvrability is the lower expense. Ship manoeuvring mathematical model-based simulation or CFD-based direct simulation are available. The main difficulty with mathematical model-based simulation is the expression of bank effect induced force/moment in the mathematical model. CFD-based direct simulation provides an effective way to calculate the hydrodynamic forces and moments of ships in restricted waters. Since the beginning of the new century, CFD technique has developed rapidly and there have been many achievements of CFD application to the prediction of ship manoeuvrability in restricted waters. In the study on bank effect on ship manoeuvrability, Lo et al. [7] applied CFD to simulate the unsteady motion of ships and analyzed the influence of ship speed and ship-bank distance on yaw angle and transverse force. Wang [8] studied the viscous flow field of several ship types in shallow water and shallow narrow channels during oblique motion, rotary motion, and near bank navigation. Zou et al. $[9,10]$ addressed the hull squat phenomenon of ships sailing near the bank in shallow water. Zhang et al. [11, 12] analyzed the hydrodynamic characteristics of KCS container ships sailing along sloping river banks.

Although CFD has presented its powerful calculation ability in ship manoeuvring hydrodynamics, the verification and validation $(\mathrm{V} \& \mathrm{~V})$ of this method is of great importance to confirm the feasibility of this kind of numerical prediction method, which is also the purpose of series of international workshops SIMMAN 2008, 2014, and coming 2021. In 1997, Coleman and Stern [13] introduced the uncertainty analysis to ship CFD. In 2002, International Towing Tank Conference (ITTC) recommended the corresponding procedure for the V\&V of CFD [14]. During the last decade, the uncertainty analysis of ship CFD has been increasingly paid attention to. Simonsen and Stern [15] addressed the V\&V of RANS maneuvering simulation of tanker Esso Osaka. Kim et al. [16] conducted V\&V of RANS simulation of a submarine SUBOFF. Tahara et al. [17] evaluated CFD as a tool for KCS hull form design along with application of two RANS equation solvers. Uncertainty analysis in CFD for resistance and flow field was performed by Zhang et al. [18]. Zou et al. performed the $\mathrm{V} \& \mathrm{~V}$ of numerical predictions of hydrodynamics on KVLCC2 ship in shallow waters [19] and narrow waters [20]. Yang et al. [21] presented the results of uncertainty analysis for resistance and wave profile of KCS computed by RANS and DES methods was verified and validated. Zhu et al. [22] performed CFD uncertainty analysis for simulation of roll motions for a 3D ship DTMB 5512. Simone et al. [23] presented a numerical roll damping assessment of the intact DTMB 5415 naval ship at zero speed. Islam and Soares [24] performed an uncertainty analysis by using OpenFOAM for four different ship models: KCS, DTC, KVLCC2, and JBC. Hrvoje et al. [25] presented the CFD validation and grid sensitivity studies on full-scale ship self-propulsion.
Generally speaking, the V\&V of CFD in ship manoeuvring is a hot topic in the community of ship hydrodynamics. Nevertheless, to improve the feasibility of CFD in the prediction of ship manoeuvrability, some issues need to be further addressed. Firstly, more study should be performed with respect to unstructured grids since the present procedure recommended by ITTC mainly focuses on structured grids. Secondly, even though structured grids are adopted, how to define the refinement rate of grids in the boundary layer needs to be further studied. Inappropriate refinement rate might make the near-wall grids unmatched with turbulence model. As a result, both the calculation accuracy and convergence cannot be guaranteed. In the study, unstructured grids are used and the global element scale factor is taken as the same as refinement ratio to control the number of grids. Grid independence analysis is conducted after the unstructured grids are generated. Corresponding CFD uncertainty analysis is conducted according to the procedure recommended by ITTC. The hydrodynamic forces of KVLCC2 ship sailing near inclined banks are calculated based on RANS equations. RNG turbulence model is adopted. Direct and oblique navigation conditions near inclined banks are investigated. In direct navigation case, the variation of transverse force and yaw moment of KVLCC2 hull with the ship-shore distance, bank angle, and water depth are analyzed. Also the transverse force and yaw moment of hull-propeller-rudder model are calculated. In oblique navigation case, the variation of transverse force and yaw moment with the bank angle, water depth, and drift angle is analyzed.

\section{Physical Problems and Numerical Methods}

2.1. Problem Description. As shown in Figure 1, a ship is sailing in the vicinity of a bank. The bank slope is constant with angle $\theta$ along the longitudinal direction. The rectangular coordinate system $o-x y z$ is attached to the ship and the Earth-fixed coordinate system is $o^{\prime}-x^{\prime} y^{\prime} z^{\prime}$. The origin of attached coordinate system is located at the center of gravity. $h$ is the water depth w.r.t. the longitudinal section in center plane. $\eta$ is the ship-shore distance.

2.2. Governing Equation. For incompressible fluid, the continuity equation and N-S equation can be described as

$$
\begin{gathered}
\nabla \cdot \mathbf{u}=0 \\
\frac{\partial \mathbf{u}}{\partial t}+(\mathbf{u} \nabla) \mathbf{u}=-\frac{1}{\rho} \nabla p+\nu \Delta \mathbf{u},
\end{gathered}
$$

where $\nabla$ is the Nabla operator; $\Delta$ is the Laplace operator; $\mathbf{u}=(u, v, w)$ is the fluid velocity vector; $p$ is the pressure; $\rho$ is the density of the fluid; and $v$ is the viscosity coefficient of fluid movement. The Reynolds average method is used to decompose the variables in the above equation into the sum of the time-averaged component and the fluctuating component. Then, the continuity equation and N-S equation become the following equations in terms of tensor: 


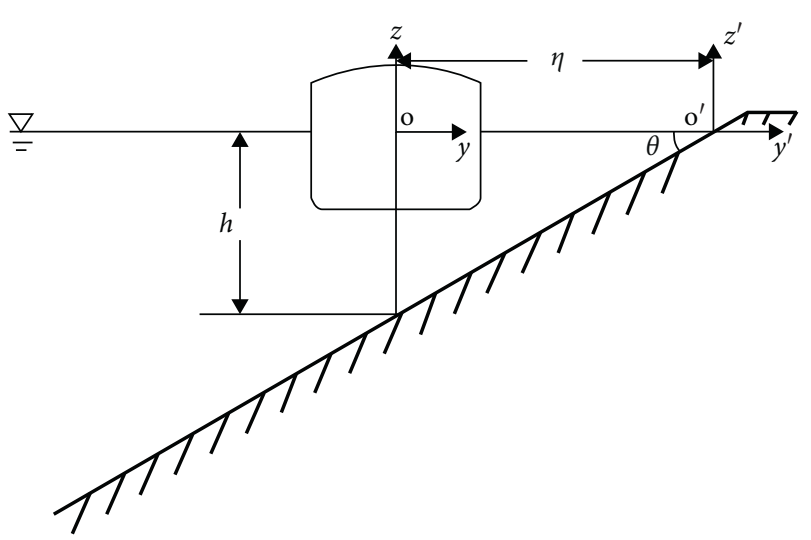

Figure 1: Coordinate systems.

$$
\begin{gathered}
\frac{\partial u_{i}}{\partial x_{i}}=0 \\
\rho \frac{\partial u_{i}}{\partial t}+\rho u_{j} \frac{\partial u_{i}}{\partial x_{j}}=-\frac{\partial p}{\partial x_{i}}+\frac{\partial}{\partial x_{j}}\left(\rho \nu \frac{\partial u_{i}}{\partial x_{j}}-\overline{\rho u_{i}^{\prime} u_{j}^{\prime}}\right),
\end{gathered}
$$

where $-\overline{\rho u_{i}^{\prime} u_{j}^{\prime}}$ is the Reynolds stress term. Equation (3) is the so-called RANS equation, which needs to be closed by adding a turbulence model.

2.3. Turbulence Model. Two kinds of two-equation turbulence model are in common use, i.e., the $k-\varepsilon$ model and $k-\omega$ model. In the study, the RNG $k-\varepsilon$ turbulence model is employed, which is derived by a statistical method called Renormalization Group Theory. Compared with the standard $k-\varepsilon$ model, the RNG $k-\varepsilon$ turbulence model gains better adaptability, accuracy, and credibility since it corrects the turbulent viscosity and considers the rotation and swirling flow in the average flow. After adding an additional term to the $\varepsilon$-equation, the RNG $k-\varepsilon$ model can reflect the time average strain rate of main flow and the spatial coordinate function to improve the calculation accuracy of rapid deformation flow as well.

The transportation equation of RNG $k-\varepsilon$ turbulence model is as follows:

$$
\begin{aligned}
\frac{\partial}{\partial t}(\rho k)+\frac{\partial}{\partial x_{i}}\left(\rho k u_{i}\right)= & \frac{\partial}{\partial x_{j}}\left[\left(\mu+\frac{\mu_{t}}{\sigma_{k}}\right) \frac{\partial k}{\partial x_{j}}\right]+G_{K}-\rho \varepsilon \\
\frac{\partial}{\partial t}(\rho \varepsilon)+\frac{\partial}{\partial x_{i}}\left(\rho \varepsilon u_{i}\right)= & \frac{\partial}{\partial x_{j}}\left[\left(\mu+\frac{\mu_{t}}{\sigma_{\varepsilon}}\right) \frac{\partial \varepsilon}{\partial x_{j}}\right] \\
& +C_{1 \varepsilon} G_{K} \frac{\varepsilon}{k}-C_{2 \varepsilon} \rho \frac{\varepsilon^{2}}{k}-R_{\varepsilon}
\end{aligned}
$$

where $k$ is the turbulent kinetic energy; $\mu$ and $\mu_{t}$ are the dynamic viscosity and turbulent viscosity, respectively; $G_{K}$ is a production of turbulent kinetic energy; $\sigma_{k}, \sigma_{\varepsilon} C_{1 \varepsilon}, C_{2 \varepsilon}$ are model constants; $\varepsilon$ is the turbulence dissipation; and the additional term $R_{\varepsilon}$ is calculated as

$$
R_{\varepsilon}=\frac{C_{\mu} \rho \eta^{3}\left(1-\eta / \eta_{0}\right)}{1+\beta \eta^{3}} \cdot \frac{\varepsilon^{2}}{k},
$$

where $\eta_{0}, C_{\mu}$, and $\beta$ are constants and $\eta$ and $S_{i j}$ are determined by

$$
\begin{gathered}
\eta=\sqrt{2 S_{i j} S_{i j}} \frac{k}{\varepsilon} \\
S_{i j}=\frac{1}{2}\left(\frac{\partial u_{i}}{\partial x_{j}}+\frac{\partial u_{j}}{\partial x_{i}}\right) .
\end{gathered}
$$

2.4. Computation Domain and Boundary Condition. KVLCC2 ship model is selected as the investigated object, which is one of the benchmark ship models recommended by ITTC, as shown in Figure 2. To validate the CFD calculation results by comparing with experimental data, the ship model in CFD calculation is also taken as the same as provided by INSEAN (the towing tank in Rome, Italy). The main particulars of the ship model are shown in Table 1. The speed of the vessel is $U=0.533 \mathrm{~m} / \mathrm{s}$, with length-based Froude number $F_{r}=0.0643$.

Figure 3 presents the computation domain and corresponding boundary condition settings.

(1) Inlet. The calculation area starts from the location with a distance of one ship's length upstream of the bow and is set as the velocity inlet boundary; the specific turbulence parameters include the turbulence intensity and turbulence viscosity ratio, with the values $2 \%$ and 2 , respectively.

(2) Outlet. The calculation area ends at the location with a distance of 2 times the ship's length downstream of the stern and is set as outflow boundary; it is assumed that the flow is fully developed at outlet.

(3) Free Surface. Due to the low speed of the ship $\left(F_{r}=0.0643\right)$, the effect of wave making on the free surface can be ignored. Therefore, it is set as the boundary of symmetry surface.

(4) Surface Boundary. Both the hull surface and the bottom of channel are set as no-slip wall condition.

(5) Other Boundaries. The vertical plane on the left side of the ship is located one time the length of the ship, which is also set as the symmetric boundary condition.

2.5. Computational Grid. In the study, a kind of unstructured grid, tetrahedral grid, is used in the whole fluid domain. The grids near the hull and in the stern area are densified. The height of the first-level grid node of the boundary layer grid is appropriately set so that $y^{+}$falls within the range of $[30,300]$. Consequently, the wall function method is used to simulate the near-wall boundary layer flow situation. Figure 4 presents the generation of grids around the ship on the undisturbed free surface. 


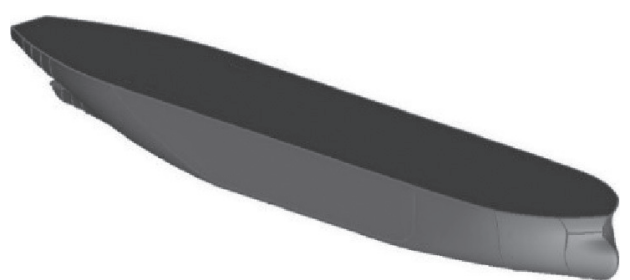

FIgUre 2: Profile of the KVLCC2 ship.

TABLE 1: Main particulars of the KVLCC2 ship.

\begin{tabular}{lccc}
\hline Item & Symbols & Full scale & Model scale \\
\hline Scale ratio & & $1: 1$ & $1: 45.714$ \\
Perpendicular length & $L_{p p}(\mathrm{~m})$ & 320 & 7 \\
Waterline length & $L_{w l}(\mathrm{~m})$ & 325.5 & 7.1204 \\
Breadth & $B(\mathrm{~m})$ & 58 & 1.269 \\
Draft & $T(\mathrm{~m})$ & 20.8 & 0.455 \\
Breadth coefficient & $C_{B}$ & 0.8098 & 0.8098 \\
Displacement & $\nabla\left(\mathrm{m}^{3}\right)$ & 312622 & 3.2724 \\
Wetted surface area & $S\left(\mathrm{~m}^{2}\right)$ & 27320 & 13.0129 \\
\hline
\end{tabular}

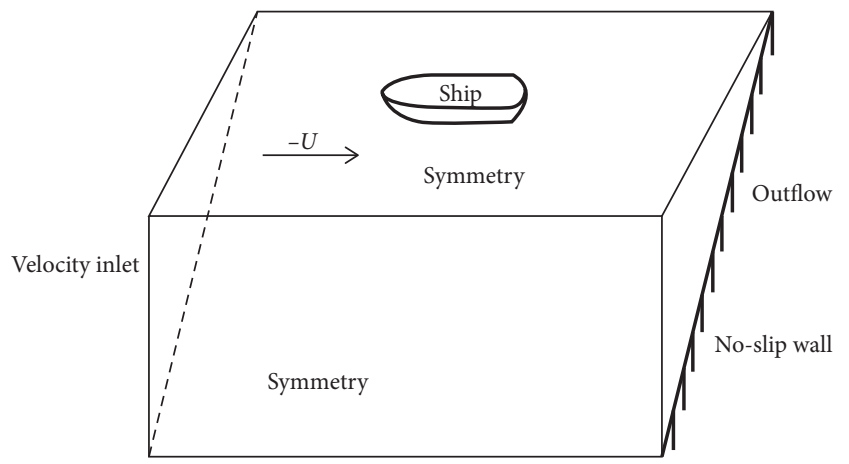

FIgURE 3: Computation domain and boundary.

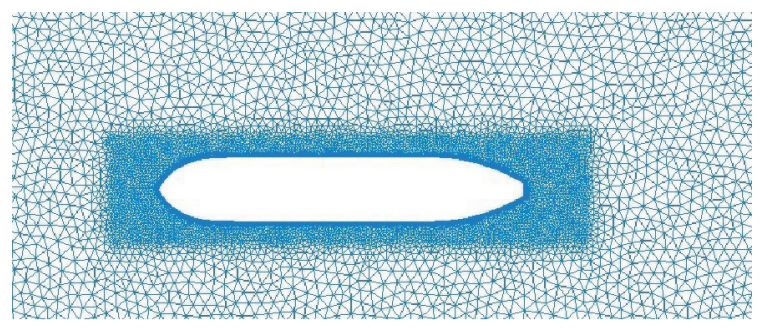

FIGURE 4: Grids on the undisturbed free surface.

2.6. Numerical Method. The commercial solver FLUENT is used for calculation, in which the finite volume method is utilized to discrete governing equations. The speed-pressure coupling equation is calculated by using the pressure-based separation algorithm SIMPLEC. To obtain high numerical accuracy, the second-order upwind scheme is used for momentum, turbulent kinetic energy, and turbulent dissipation rate. The underrelaxation factor is taken as the default value.

\section{Verification and Validation of the Numerical Method}

To verify the numerical method in the study, the hydrodynamics of KVLCC2 ship model sailing at a constant forward speed with $U=0.533 \mathrm{~m} / \mathrm{s}$ in shallow water with $h=$ $1.5 T$ (where $h$ is water depth and $T$ is draft; corresponding depth-based Froude number $F_{H}=0.206$ ) are calculated and compared with the test data [26]. Three sets of grids are adopted, respectively: coarse, medium, and fine. The mesh refinement ratio is set as $\sqrt{2}$, as recommended by ITTC. Global element scale factor is set as the same value to control the number of grids.

3.1. Verification. According to the procedure recommended by ITTC, the numerical uncertainty of ship viscous flow calculation $U_{\mathrm{SN}}$ consists of iterative uncertainty $U_{I}$ and grid uncertainty $U_{G}[14]$ :

$$
U_{\mathrm{SN}}=\sqrt{U_{G}^{2}+U_{I}^{2}}
$$

The iterative uncertainty is determined by [27]

$$
U_{I}=L_{\infty}(\Delta \phi)=\max \left(\left|\Delta \phi_{i}\right|\right), \quad 1 \leq i \leq N_{P},
$$

where $N_{P}$ represents the number of grid nodes and $\Delta \phi$ is the local change of the flow quantity. Table 2 lists the comparison of iteration with respect to coarse, medium, and fine grids. $S_{1}, S_{2}$, and $S_{3}$ stands for coarse, medium, and fine grids, respectively.

The convergence of iteration is mainly judged by the variation of iterative residual. Usually, a reduction of the residuals by 3 orders of magnitude after iteration indicates at least qualitative convergence. Figure 5 presents the iteration history by using medium grids. As can be seen, from the initialization of the flow field to the end of iteration, the residuals are reduced by 3 to 4 orders of magnitude compared to the initial iteration.

To analyze the grid uncertainty $U_{G}$, a metric convergence rate $R_{G}$ is firstly defined as

$$
R_{G}=\frac{\varepsilon_{G 23}}{\varepsilon_{G 12}},
$$

where $\varepsilon_{G 12}$ denotes the difference between numerical results of coarse and medium grids while $\varepsilon_{G 23}$ denotes the difference between numerical results of medium and fine grids. Grid convergence depends on the value of $R_{G}$ in three cases: (1) $R_{G}<0$, oscillation convergence; (2) $0<R_{G}<1$, monotonic convergence; and (3) $R_{G}>1$, divergence. Based on the calculation results presented in Table 2 , the convergence rate $R_{G}$ can be calculated as $R_{G}=0.6578$, which means the monotonic convergence. By defining the order of accuracy

$$
P_{G}=\frac{\ln R_{G}^{-1}}{\ln r_{G}},
$$

and estimated numerical error

$$
\delta_{\mathrm{RE}_{G}}^{*}=\frac{\varepsilon_{G 23}}{r_{G}^{P_{G}}-1},
$$


TABLE 2: Iterative uncertainty analysis in resistance calculation by using three sets of grid.

\begin{tabular}{lcccc}
\hline Item & $\begin{array}{c}\text { Coarse } \\
\left(S_{1}\right)\end{array}$ & $\begin{array}{c}\text { Medium } \\
\left(S_{2}\right)\end{array}$ & Fine $\left(S_{3}\right)$ & $\begin{array}{c}\text { Test result } \\
(\mathrm{S})\end{array}$ \\
\hline Grid & 897231 & 1689704 & 3076847 & \\
quantity & -0.028926 & -0.028362 & -0.027991 & -0.0286 \\
$X^{\prime}$ & $2.5 \times 10^{-4}$ & $6.7 \times 10^{-4}$ & $7.5 \times 10^{-4}$ & \\
\hline$U_{I}\left(\% X^{\prime}\right)$ &
\end{tabular}

$X^{\prime}$ stands for the nondimensional resistance.
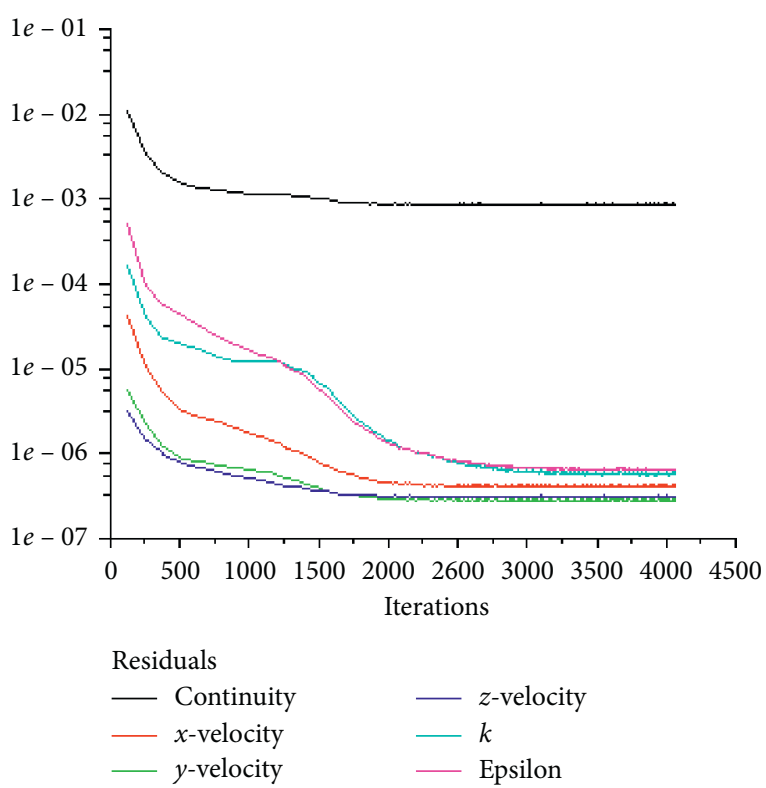

Figure 5: Histories of residuals.

where $r_{G}$ and $r_{G}^{P_{G}}$ are refinement ratios, also by using correction factor $C_{G}$, the grid uncertainty $U_{G}$ can be calculated as

$$
U_{G}=\left|C_{G} \delta_{\mathrm{RE}_{G}}^{*}\right|+\left|\left(1-C_{G}\right) \delta_{\mathrm{RE}_{G}}^{*}\right|,
$$

when solutions are far from the asymptotic range, i.e., $C_{G}$ is sufficiently less than or greater than 1 ; or $U_{G}$ can be calculated by

$$
U_{G_{C}}=\left|\left(1-C_{G}\right) \delta_{\mathrm{RE}_{G}}^{*}\right|,
$$

when solutions are close to the asymptotic range, i.e., $C_{G}$ is close to 1 . Based on the results in Table 2, corresponding results of grid uncertainty analysis can be obtained as listed in Table 3. As can be recognized from the comparison between Tables 2 and 3, the iterative uncertainty $U_{I}$ is much less than the grid uncertainty $U_{G}$ by 4 orders, which means the numerical uncertainty of calculation mainly derives from grid uncertainty $U_{G}$, i.e., $U_{\mathrm{SN}} \approx U_{G}$ according to equation (7).

3.2. Validation. Validation of numerical calculation is mainly accomplished by comparing the error $E$ with validation uncertainty $U_{V}$. Error $E$ is defined as

$$
E=D-S \text {. }
$$

where $D$ is the experimental result and $S$ is the numerical result. It is noted that results of $D$ and $S$ have been presented in Table 2.

The validation uncertainty $U_{V}$ is calculated as

$$
U_{V}=\sqrt{U_{\mathrm{SN}}^{2}+U_{D}^{2}}
$$

where $U_{D}$ is the known measurement uncertainty. In the study, $U_{D}=6.4 \% D$. Table 4 lists the validation results by three sets of grids, i.e., coarse, medium, and fine grids. As can be recognized, $|E|<U_{V}$ holds for three sets of grids, which means validation is achieved at $U_{V}=6.87 \% D$ level.

From the above verification and validation analysis, it can be confirmed that the numerical method used in the study is valid in calculating the resistance of KVLCC2. Furthermore, as can be recognized from Table 4, the selection of medium grid gains better accuracy over the other two. Therefore, this kind of grid is used in the next calculation of manoeuvring hydrodynamics in the case of straightforward moving in the vicinity of inclined banks.

\section{Case Study}

4.1. Straightforward Movement. It is assumed that the KVLCC2 ship model is sailing at a constant forward speed with $U=0.533 \mathrm{~m} / \mathrm{s}$ in parallel to the shoreline. The dimensionless transverse force and yaw moment are defined as

$$
\begin{aligned}
Y^{\prime} & =\frac{Y}{0.5 \rho U^{2} L T}, \\
N^{\prime} & =\frac{N}{0.5 \rho U^{2} L^{2} T},
\end{aligned}
$$

where $L$ is taken as the perpendicular length, i.e., $L_{p p}$.

First of all, hull is only considered in the manoeuvring simulation. Figure 6 shows the variation of transverse force with different ship-bank distances $\eta$ (in dimensionless form $\left.\eta B^{-1}\right)$. As can be seen, the transverse force points to the inclined river bank, which means "bank suction" happens. This phenomenon can be explained according to Bernoulli's equation: the streamlines on the right side of the ship become dense so that the pressure on the right side of the ship is lower than that on the left side, which leads to the transverse force on the ship pointing to the shore wall. It is also noted that under the same ship-bank distance, the transverse force decreases with an increase of the inclination of the bank wall. This is because under the same ship-bank distance, the bigger the inclination of the bank is, the larger the area of the cross section on the right side of the ship is, which implies that the flow velocity becomes smaller according to the continuity equation. As a result, the pressure on the right side of the ship in the case of a larger inclination is larger than the case of a smaller inclination. Under the same pressure on the left side of ship, the pressure difference decreases with the increase of inclination. Figure 6 also indicates the common trend of vanishing transverse 
TABLE 3: Grid uncertainty analysis in resistance calculation.

\begin{tabular}{lcccc}
\hline Item & $R_{G}$ & $P_{G}$ & $C_{G}$ & $U_{G}(\% S)$ \\
\hline$X^{\prime}$ & 0.6578 & 1.21 & 0.521 & 2.49 \\
\hline
\end{tabular}

TABLE 4: Validation analysis of resistance calculation by using three sets of grid.

\begin{tabular}{lccc}
\hline Item & Coarse $\left(S_{1}\right)$ & Medium $\left(S_{2}\right)$ & Fine $\left(S_{3}\right)$ \\
\hline$E(\% D)$ & -1.14 & 0.83 & 2.13 \\
$U_{V}(\% D)$ & 6.87 & 6.87 & 6.87 \\
\hline
\end{tabular}

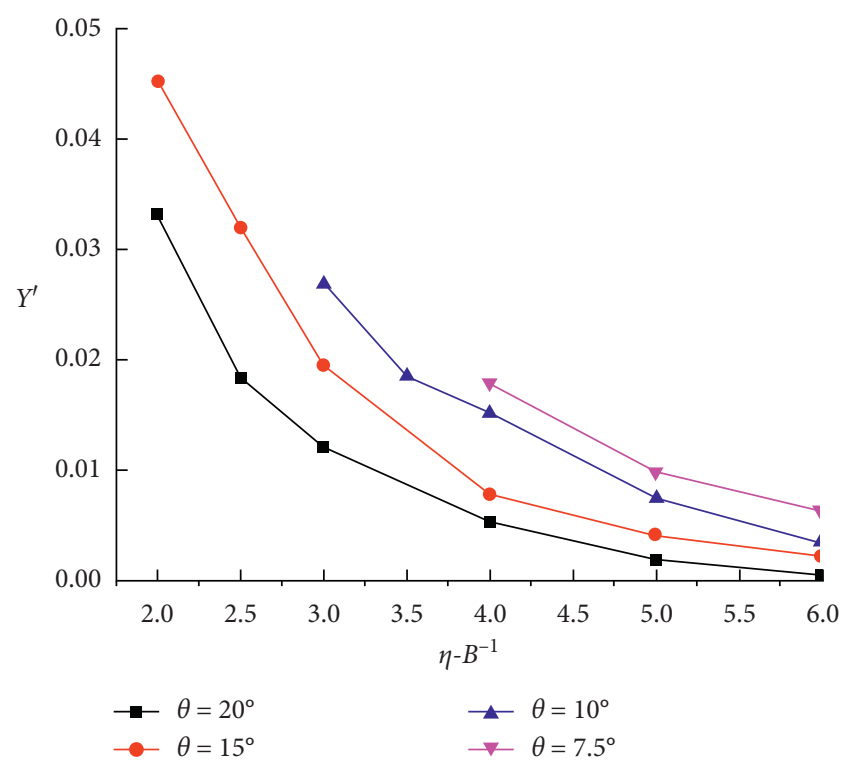

Figure 6: Variation of transverse force with different ship-bank distances.

force (or the bank suction effect) with the increase of shipbank distance.

Figure 7 shows the change of transverse force with the increase of water depth in dimensionless form $h T^{-1}$. As can be recognized, with the increase of water depth, the transverse force on the ship decreases generally. This is because the increase of water depth with a fixed inclination angle of bank implies the increase of ship-bank distance. It can also be seen that with the increase of the inclination angle, the bank suction effect is gradually increasing. This is because the increase of the inclination angle of bank with a fixed water depth implies the decrease of ship-bank distance. As a result, the effect of bank suction amplifies.

Figure 8 shows the variation of yaw moment with the ship-bank distance. It can be seen that in all cases the yaw moments become smaller with the increase of the ship-bank distance. This is because the flow area on the right side of the ship becomes larger with the increase of the ship-bank distance. As a result, the reflection effect of the shore wall decreases. It can be also recognized that the phenomenon bow-out happens to KVLCC2, which means the bow is turning off the bank [28]. This can be illustrated from the pressure distribution around the bow and stern areas as shown in Figure 9, for instance, $\theta=15^{\circ}, \eta=2.5$. Obviously, the high pressure at bow near bank versus the low pressure at stern near bank leads to the bow-out effect.

Figure 10 shows the variation of yaw moment with water depth. It can be seen that at the same water depth, the larger the inclination angle of bank is, the larger the yaw moment is. This is because the increase of the inclination angle of bank with a fixed water depth implies the decrease of shipbank distance, as aforementioned.

4.2. Oblique Movement. As shown in Figure 11, in the case of oblique movement, the ship is moving at a cruising speed with a fixed drift angle $\beta$ (positive as shown in Figure 11). The shore locates on the right side of the ship. It should be noted that in the given coordinate system a positive yaw moment pushes the bow away from the bank, or points to the positive direction of $Z$-axis if the yaw moment is defined as a vector.

$\mathrm{V} \& \mathrm{~V}$ is first conducted for the confirmation of iteration and grid convergence w.r.t. the numerical calculation of transverse force and yaw moment. According to the test results, in simulation, the cruising speed of vessel is set as $U=0.533 \mathrm{~m} / \mathrm{s}$; drift angle is $\beta=4^{\circ}$; and water depth is $h=1.5 T$. Table 5 lists the iterative uncertainty analysis by using three sets of grid. In a similar way as the work in the case of straightforward moving, global element scale factor is used to control the number of grids and taken as the same value as refinement rate, i.e., $\sqrt{2}$. Based on the results in Table 5, the grid uncertainty analysis is presented in Table 6 . As can be recognized, $0<R_{G}<1$ holds, which means the monotonic convergence of the iteration. Moreover, the iterative uncertainty $U_{I}$ is much less than the grid uncertainty $U_{G}$ by 4 orders, which means the numerical uncertainty of calculation mainly derives from grid uncertainty $U_{G}$, i.e., $U_{\mathrm{SN}} \approx U_{G}$.

Table 7 lists the validation results by three sets of grids, i.e., coarse, medium, and fine grids. The measurement uncertainty is known as $2.8 \% D$ for transverse force while it is $3.6 \% D$ for yaw moment. As can be recognized, $|E|<U_{V}$ holds for three sets of grids, which means validation for transverse force is achieved at the $U_{V}=4.61 \% D$ level while for yaw moment at $U_{V}=4.0 \% D$ level.

From the above V\&V analysis, it can be confirmed that the numerical method used in the study is valid in simulating the manoeuvring of KVLCC2. Furthermore, as can be recognized from Table 7, the selection of medium grid gains better accuracy over the other two. Therefore, this kind of grid is used in the calculation of hydrodynamics in the case of oblique moving near inclined banks. Figure 12 presents the variation of manoeuvring hydrodynamics with the water depth at different drift angle. The inclination angle of bank is set as $\theta=15^{\circ}$. As can be seen, both transverse force and yaw moment increase with the decrease of water depth due to the increasing bank effect since for a fixed inclination angle of bank, the decrease of water depth implies approaching the bank. It can also be seen from Figure 12 that the yaw moment increases with the increase of drift angle. Moreover, 


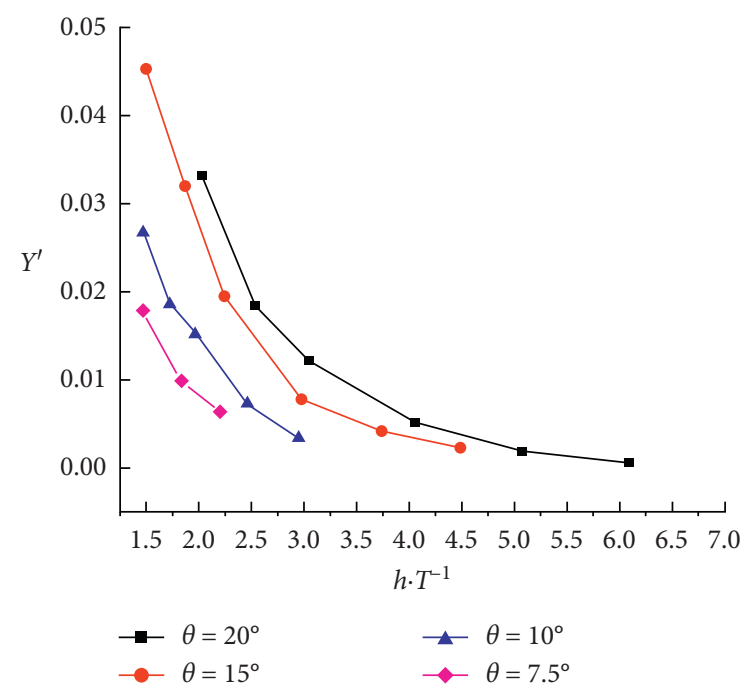

Figure 7: Variation of transverse force with different water depths.

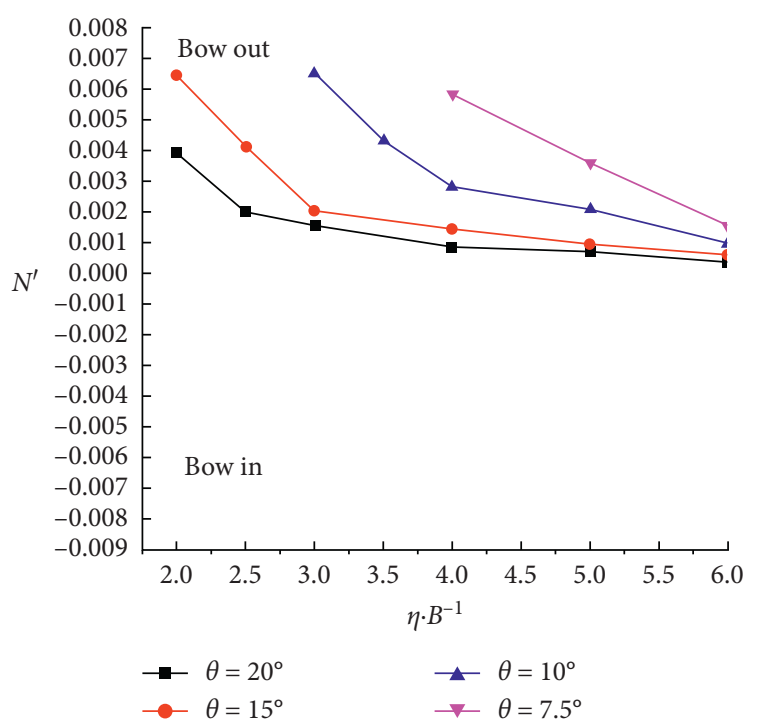

FIGURE 8: Variation of yaw moment with different ship-bank distances.

the direction of yaw moment changes with the change of direction of drift angle. If the drift angle is positive (as shown in Figure 12), the streamlines along the right side of the bow are denser than stern. As a result, the pressure difference (pointing to the bank) at the bow is larger than stern, which produces a moment pushing the bow towards the bank, i.e., a negative yaw moment. In a similar way, a positive yaw moment comes around if the drift angle is negative. Compared with yaw moment, the transverse force is less sensitive to the direction change of drift angle. Due to the bank suction, a small negative drift angle cannot lead to a negative transverse force (port side). It is noted that there is a crossing of lines in the plot of the variation of yaw moment. It is because the shallower the water depth is, the more sensitive to the drift angle the yaw moment is, which means that the change of yaw moment becomes more intense in the case of shallow water. Therefore, the slope of the regressive line in the case of shallow water is larger than deep water, which results in the crossing of lines at around $\beta=0.5^{\circ}$.

Figure 13 presents an example of the pressure distribution on free surface along the hull in the case of $\theta=$ $20^{\circ}$ and $h=2 T$. As can be seen, the effect of bank suction is obvious due to the asymmetric flow field. Moreover, the variation of the pressure difference in windward and leeward regions of the hull with the drift angle is obvious especially when the drift angle increases. For example, in the case of $\beta=2^{\circ}$, the pressure difference in the fore part between the windward (port) side and the leeward (starboard) side results in a transverse force towards the starboard side. Contrarily, the fore part of the hull in the case of $\beta=-2^{\circ}$ experiences a transverse force towards the port side.

Figure 14 presents the variation of manoeuvring hydrodynamics with inclination angle of bank at different drift angle. The water depth is set as $h=2 T$. As can be seen, both transverse force and yaw moment increase with the increase of inclination angle of bank due to the increasing bank effect since for a fixed water depth, the increase of inclination angle of bank implies approaching the bank. Similar to the variation of $Y^{\prime}$ and $N^{\prime}$ with drift angle in the case shown in Figure 12, the direction of yaw moment changes with the direction change of drift angle. On the contrary, due to the bank suction, the transverse force is less sensitive to the direction change of drift angle, compared with yaw moment. A small negative drift angle cannot lead to a negative transverse force (port side).

Figure 15 presents the variation of the pressure on the bank (opposite to the bottom of hull) with water depth in the case of $\beta=-2^{\circ}$. As can be seen, the interaction between the ship and the bank decreases with an increase of water depth. Moreover, the trend of squat and trim can be detected due to the pressure difference between the bow and stern, and the negative pressure on most parts of the ship.

4.3. Manoeuvring Simulation under Control Force. The forces induced by rudder and propeller exert an important influence on ship manoeuvring. As aforementioned, even in the case of straightforward moving, there are transverse force and yaw moment due to the bank effect. Forces and moment produced by rudder and/or propeller might counteract or alleviate the bank effect. Therefore, manoeuvring simulation is performed with respect to the hull-propeller-rudder model of KVLCC2. Main particulars of rudder and propeller are listed in Table 8.

In generating the grids for the hull-propeller-rudder model, the whole calculation domain is divided into three areas, i.e., the propeller area, stern area, and the remaining. Due to the particularity and complexity of the flow field at stern, grids are further densified, compared with the area near the surface of hull. A cylinder domain is allocated to the generation of grid for propeller. The interface is treated by using multiple reference frame (MRF) technique. The meshing at stern and propeller is shown in Figure 16. The number of grids used to generate hull, propeller, and rudder 


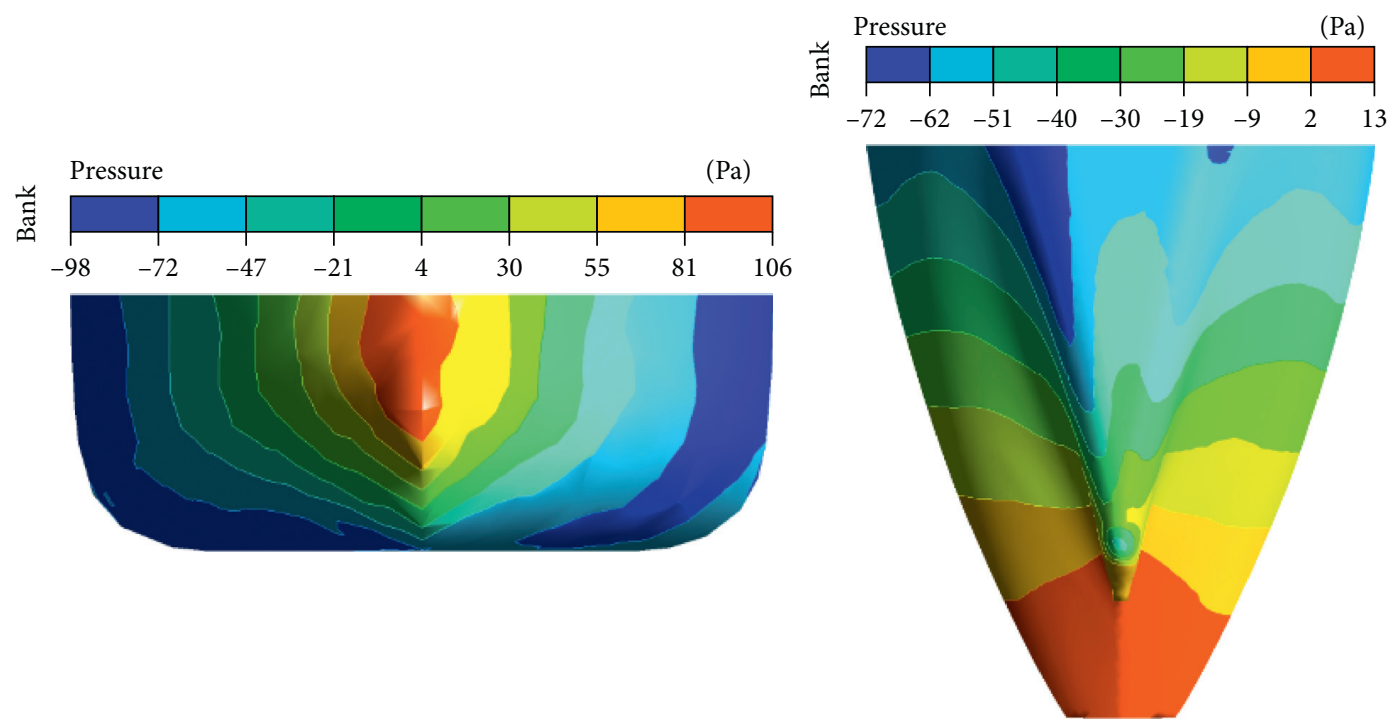

Figure 9: Pressure distribution at bow and stern at $\theta=15^{\circ}, \eta=2.5$.

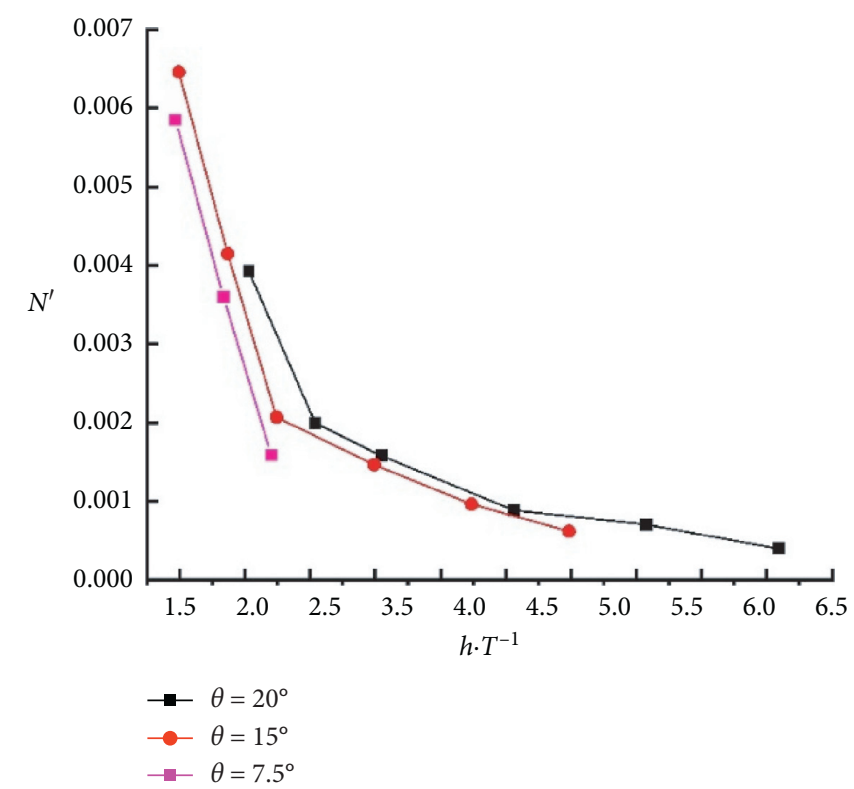

Figure 10: Variation of yaw moment with water depth.

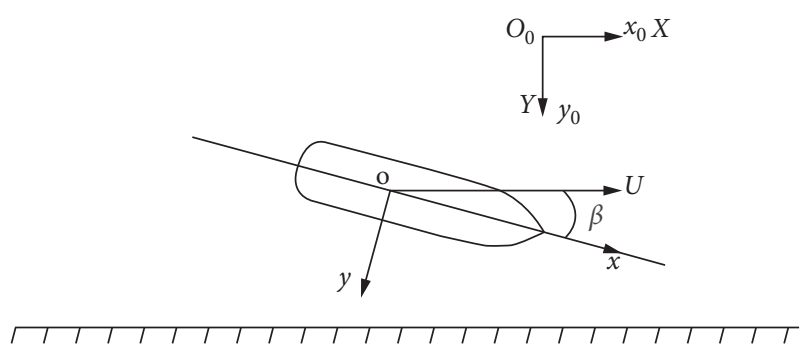

FIgURE 11: Coordinate systems for ship oblique moving.

is listed in Table 9. It is noted that there is no boundary layer mesh to be set on the surface of the rudder due to the possible degradation of the mesh quality in setting boundary layer mesh. Instead, based on the calculation formula of the height of boundary layer mesh, the mesh size of the rudder
TABLE 5: Iterative uncertainty analysis in manoeuvring simulation by using three sets of grid.

\begin{tabular}{lcccc}
\hline Item & $\begin{array}{c}\text { Coarse } \\
\left(S_{1}\right)\end{array}$ & $\begin{array}{c}\text { Medium } \\
\left(S_{2}\right)\end{array}$ & Fine $\left(S_{3}\right)$ & $\begin{array}{c}\text { Test result } \\
(S)\end{array}$ \\
\hline Grid & 1268876 & 2389602 & 4351318 & \\
quantity & & & & \\
$Y^{\prime}$ & -0.038326 & -0.037782 & -0.037391 & -0.0380 \\
$U_{I, Y^{\prime}}\left(\% Y^{\prime}\right)$ & $3.7 \times 10^{-4}$ & $5.2 \times 10^{-4}$ & $7.8 \times 10^{-4}$ & \\
$N^{\prime}$ & -0.023701 & -0.023315 & -0.023069 & -0.0234 \\
$U_{I, N^{\prime}}\left(\% N^{\prime}\right)$ & $3.3 \times 10^{-4}$ & $4.9 \times 10^{-4}$ & $5.4 \times 10^{-4}$ & \\
\hline
\end{tabular}

TABLE 6: Grid uncertainty analysis in manoeuvring simulation.

\begin{tabular}{lcccc}
\hline Item & $R_{G}$ & $P_{G}$ & $C_{G}$ & $U_{G}(\% S)$ \\
\hline$Y^{\prime}$ & 0.7188 & 0.953 & 0.391 & 3.68 \\
$N^{\prime}$ & 0.6373 & 1.30 & 0.569 & 1.84 \\
\hline
\end{tabular}


TABLE 7: Validation analysis in manoeuvring simulation by using three sets of grid.

\begin{tabular}{lccc}
\hline Item & Coarse $\left(S_{1}\right)$ & Medium $\left(S_{2}\right)$ & Fine $\left(S_{3}\right)$ \\
\hline$E_{Y^{\prime}}(\% D)$ & -0.86 & 0.57 & 1.6 \\
$U_{V, Y^{\prime}}(\% D)$ & 4.61 & 4.61 & 4.61 \\
$E_{N^{\prime}}(\% D)$ & -1.3 & 0.36 & 1.4 \\
$U_{V, N^{\prime}}(\% D)$ & 4.0 & 4.0 & 4.0 \\
\hline
\end{tabular}

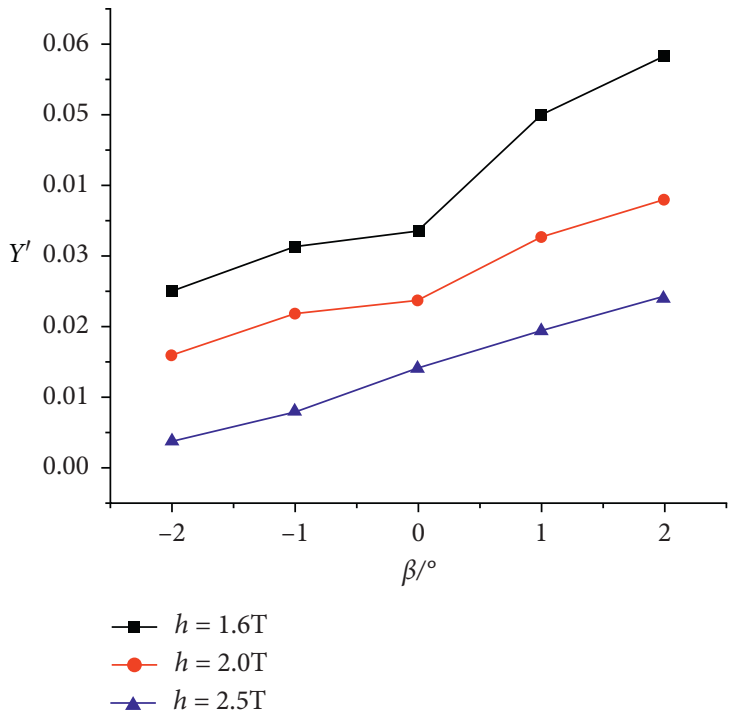

(a)

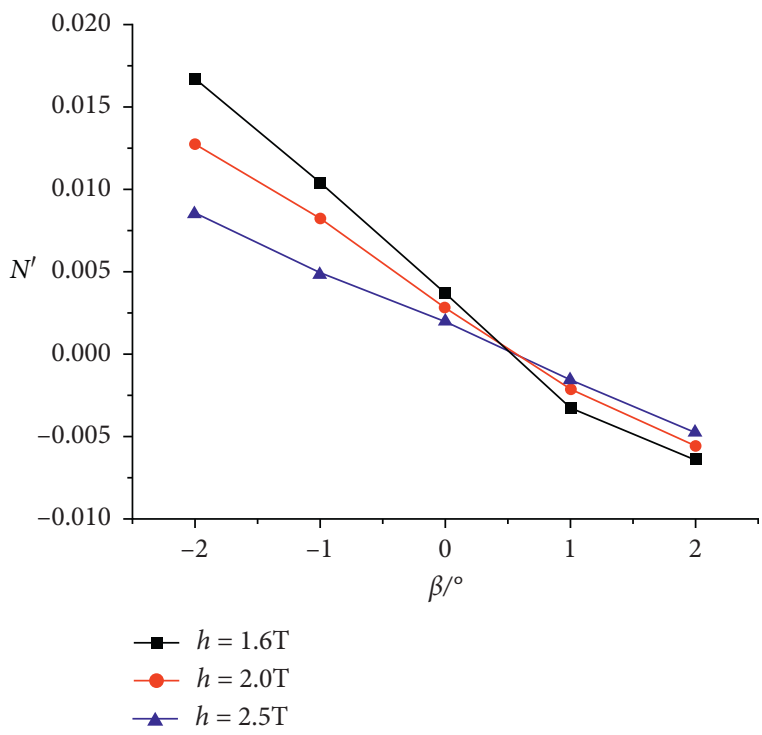

(b)

FIgURE 12: Variation of transverse force and yaw moment with water depth and drift angle.

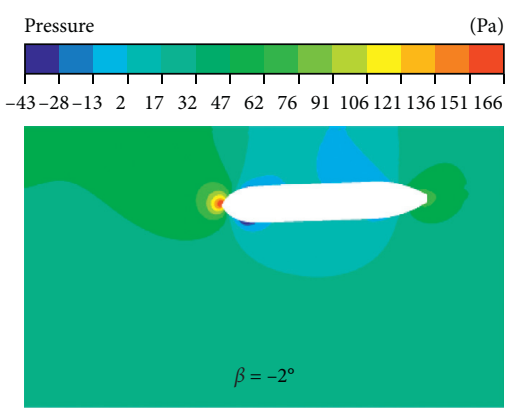

(a)

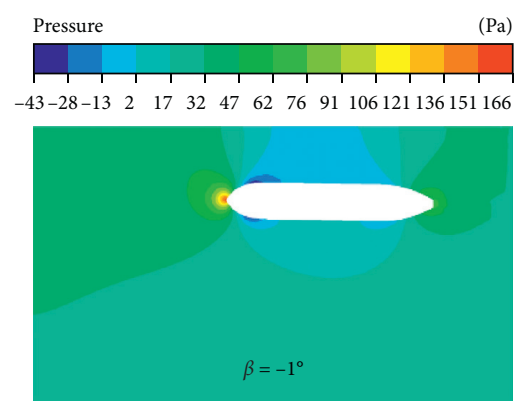

(d)

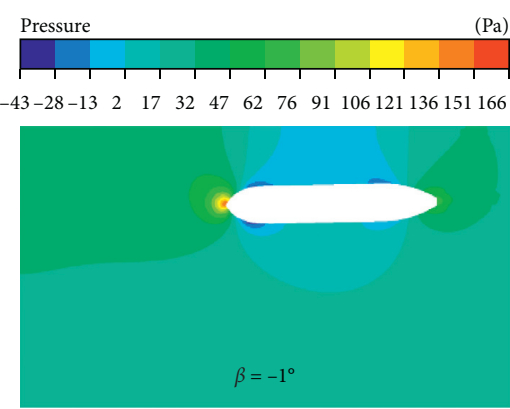

(b)

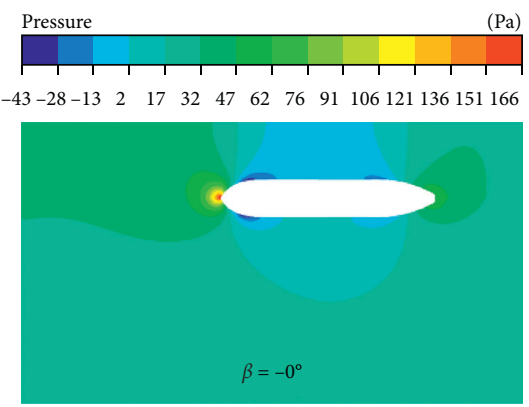

(c)

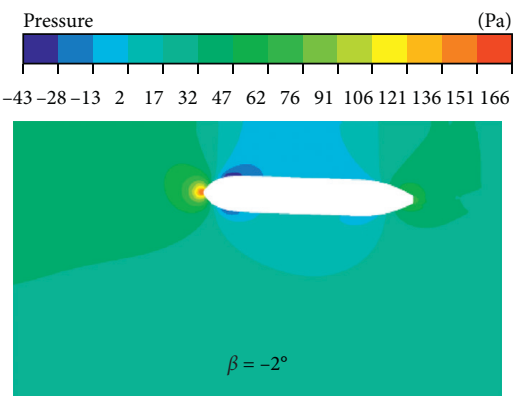

(e)

Figure 13: Variation of pressure distribution with drift angles, on free surface $\theta=20^{\circ}, h=2 T$. 


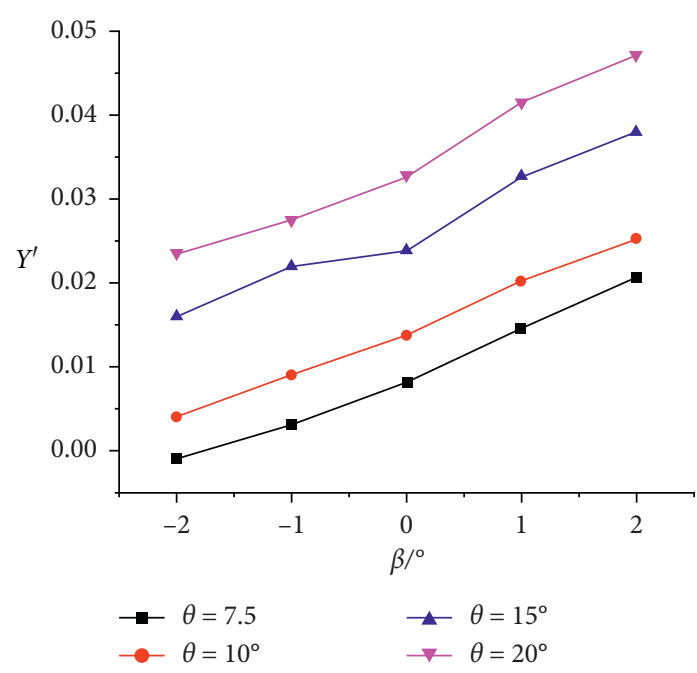

(a)

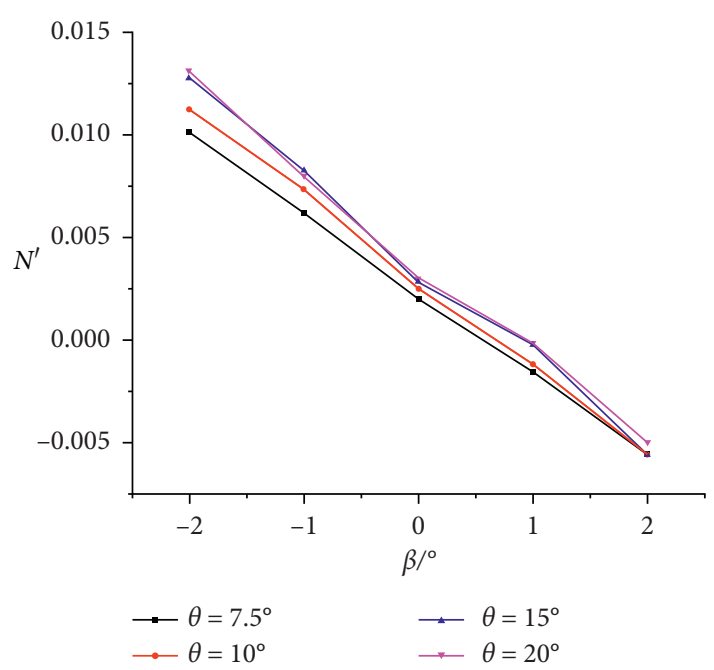

(b)

FIgURE 14: Variation of transverse force and yaw moment with inclination angle and drift angle.

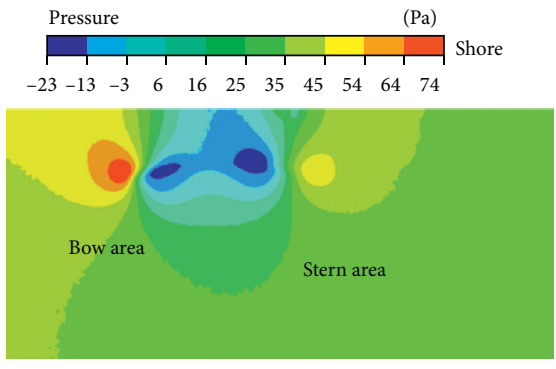

(a)

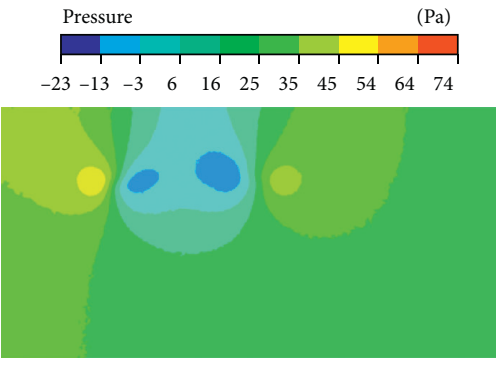

(b)

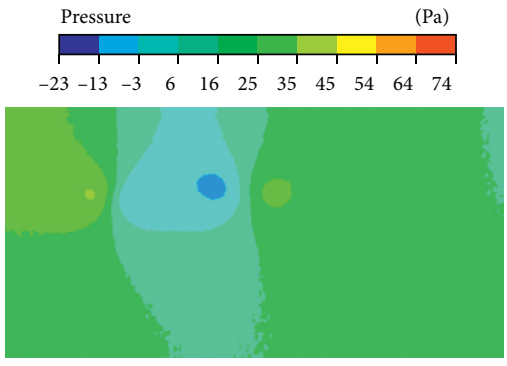

(c)

Figure 15: Variation of the pressure on the bank with water depth, $\beta=-2$. (a) $h=1.6 \mathrm{~T}$. (b) $h=2.0 \mathrm{~T}$. (c) $h=2.5 \mathrm{~T}$.

TABLE 8: Main particulars of rudder and propeller of KVLCC2.

\begin{tabular}{lc}
\hline Item & Value \\
\hline Area of rudder $\left(\mathrm{m}^{2}\right)$ & 0.1308 \\
Number of blades & 4 \\
Diameter of propeller $(\mathrm{m})$ & 0.204 \\
Pitch ratio of propeller & 0.808 \\
Area ratio of propeller & 0.448 \\
\hline
\end{tabular}

area is adjusted to ensure that the height of the first layer mesh node meets requirement.

Figures 17 and 18 present the distribution of aspect ratio of grid cells at stern and propeller, respectively, along with the check of mesh quality. As can be seen, most of the generated grids are of high quality, which verifies the effectiveness of meshing in the study.

Before manoeuvring simulation, propeller open water test is performed using CFD and compared with experiments to ensure the correct prediction of propeller forces. Table 10 and Figure 19 present the CFD calculation results and comparison results, where $J$ represents the advance ratio, $K_{T}$ is the thrust coefficient, $K_{Q}$ is the torque coefficient, and $\eta_{0}$ is the efficiency of propeller. As can be recognized from the comparison, the CFD results basically agree with the experimental results.

In manoeuvring simulation, the ship is moving straightforward at a cruising speed $U=0.533 \mathrm{~m} / \mathrm{s}$ while the revolution speed of propeller is $n=9.9 \mathrm{r} / \mathrm{s}$. The variation of thrust coefficient $\left(K_{T}\right)$ and torque coefficient $\left(K_{Q}\right)$ of propeller with rudder angle are listed in Table 11. Figure 20 presents the pressure distribution on the front of blades and back of blades, respectively, in the case of $\delta=5^{\circ}$. As can be seen, the pressure difference between the front and back is obvious for each blade, which accounts for the propulsion produced by propeller.

Figure 21 shows the variation of transverse force and yaw moment with rudder angle. In this case, the inclination angle of bank is set as $\theta=20^{\circ}$ while the initial distance between ship and shore is $\eta=2 B$.

As can be recognized, the transverse force decreases with the increase of rudder angle. Obviously, the effect of bank 


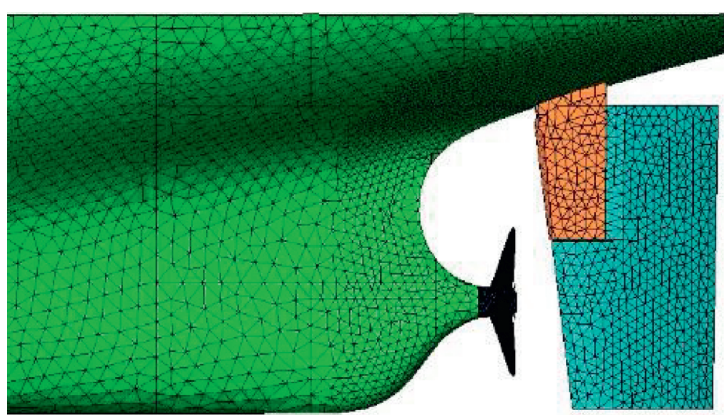

(a)

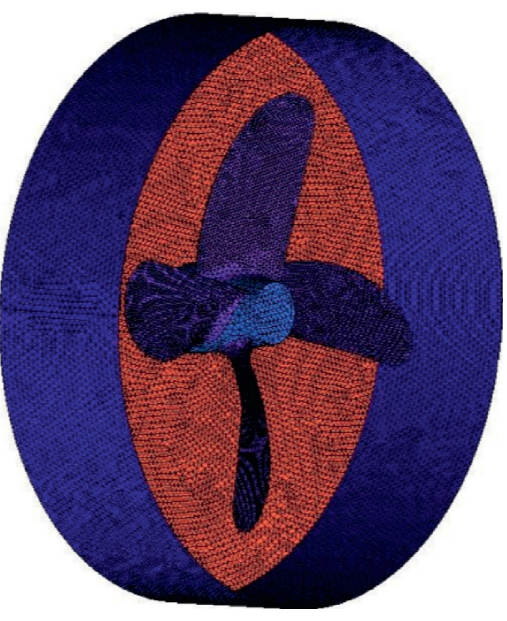

(b)

FIgURE 16: Grids at stern and propeller.

TABLE 9: Number of grids in generating hull, propeller, and rudder, respectively.

\begin{tabular}{lr}
\hline Objective & Grid quantity $\left(\times 10^{4}\right)$ \\
\hline Hull & 183.3 \\
Propeller & 37.7 \\
Rudder & 18.8 \\
\hline
\end{tabular}

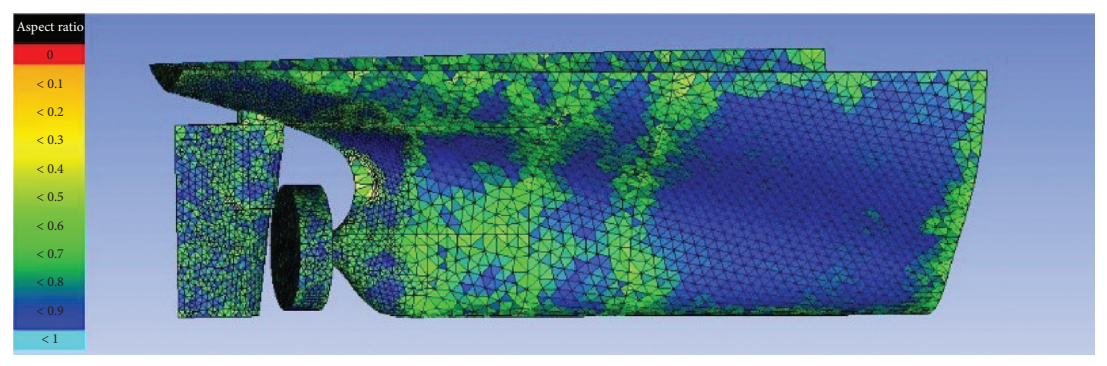

(a)

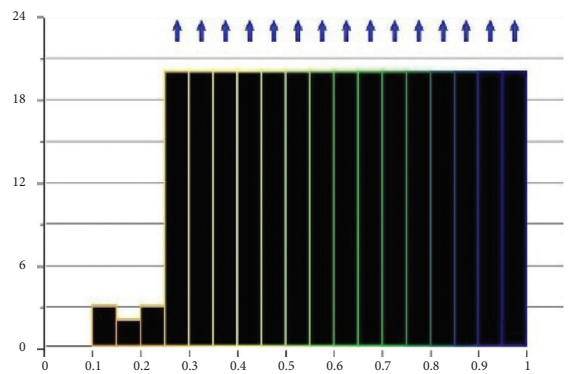

(b)

FIgURE 17: Aspect ratio of grid cells at stern and mesh quality check.

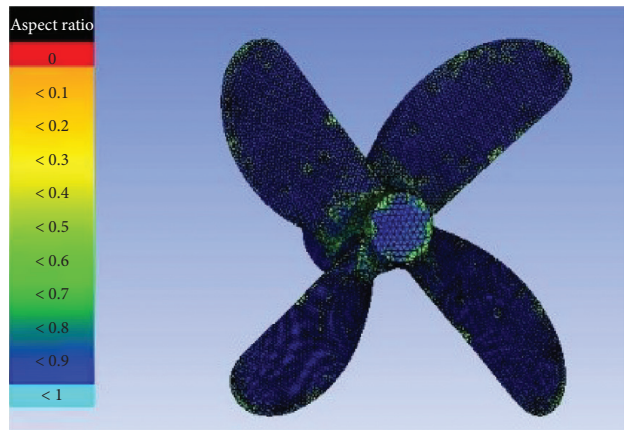

(a)

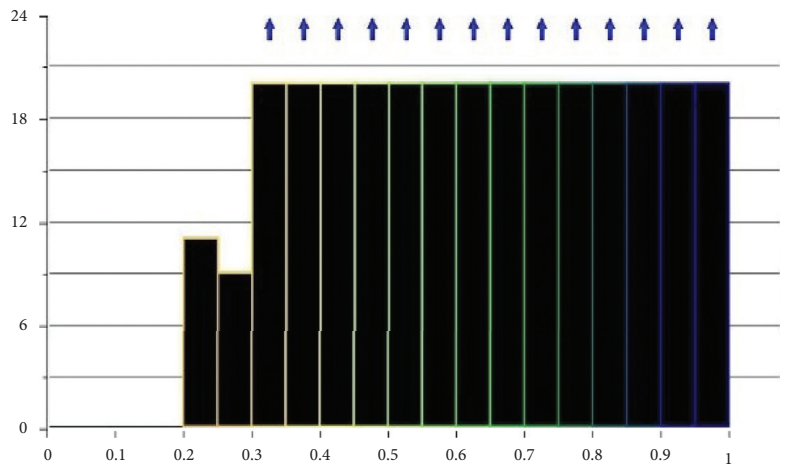

(b)

Figure 18: Aspect ratio of grid cells at propeller and mesh quality check. 
TABle 10: Propeller open water test results.

\begin{tabular}{|c|c|c|c|c|c|c|c|}
\hline \multirow{2}{*}{$J$} & \multicolumn{3}{|c|}{ Experiment } & \multicolumn{3}{|c|}{ CFD } & \multirow{2}{*}{$\begin{array}{c}\text { Error of } \\
\eta_{0}(\%)\end{array}$} \\
\hline & $K_{T}$ & $K_{Q}$ & $\eta_{0}$ & $K_{T}$ & $K_{Q}$ & $\eta_{0}$ & \\
\hline 0.1 & 0.2843 & 0.2932 & 0.1543 & 0.2518 & 0.2644 & 0.1516 & 1.74 \\
\hline 0.3 & 0.2132 & 0.2388 & 0.4263 & 0.1922 & 0.2201 & 0.4172 & 2.13 \\
\hline 0.5 & 0.1365 & 0.1721 & 0.6312 & 0.1102 & 0.1500 & 0.5849 & 7.34 \\
\hline 0.6 & 0.0951 & 0.1344 & 0.6757 & 0.0741 & 0.1098 & 0.6444 & 4.63 \\
\hline 0.7 & 0.0511 & 0.0915 & 0.6222 & 0.0479 & 0.0960 & 0.5561 & 10.6 \\
\hline 0.8 & 0.0040 & 0.0402 & 0.1267 & 0.0070 & 0.0671 & 0.1328 & 4.81 \\
\hline
\end{tabular}

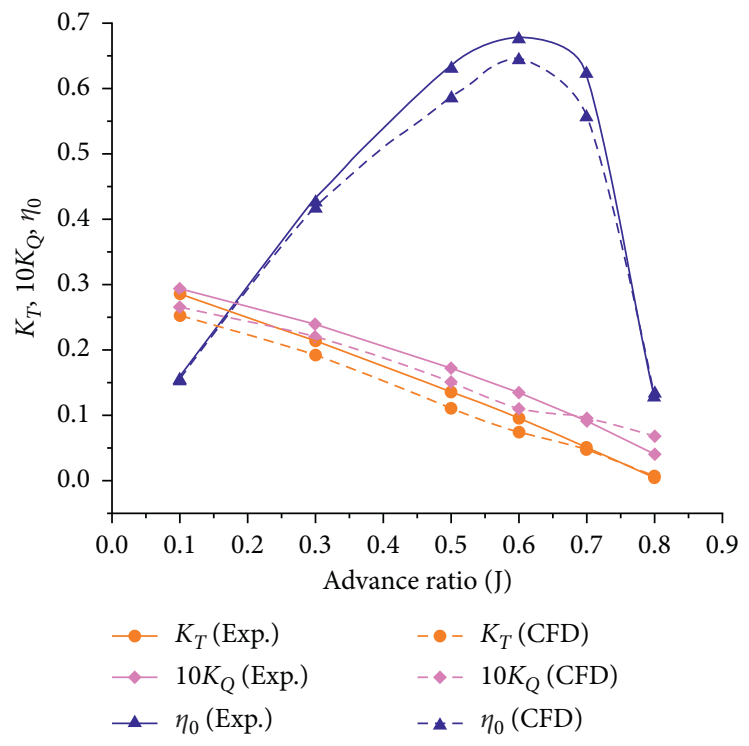

FIgURE 19: Propeller open water test results and comparison.

TABle 11: Thrust coefficient and torque coefficient of propeller.

\begin{tabular}{lcr}
\hline$\delta\left(^{\circ}\right)$ & $K_{T}$ & $10 K_{Q}$ \\
\hline 5 & 0.0368 & 0.0559 \\
10 & 0.0553 & 0.0718 \\
15 & 0.0487 & 0.0664 \\
20 & 0.0547 & 0.0714 \\
\hline
\end{tabular}

suction is alleviated due to a positive rudder angle (starboard). As for the yaw moment, on the one hand, it decreases with the increase of rudder angle (starboard), which means the effect of bow-out is alleviated. On the other hand, bow-in happens at certain rudder angle, larger than $17.8^{\circ}$ in the study. In other words, the yaw moment vanishes at $\delta=17.8^{\circ}$. Figure 22 shows the velocity distribution around propeller and rudder at stern $\left(\delta=20^{\circ}\right)$ while Figure 23 presents the vorticity distribution around propeller and rudder. As can be seen, the propeller wake deforms when reaching the rudder and affects the velocity distribution in windward and leeward regions of the rudder.

Figure 24 presents the distribution and variation of pressure on the surface of rudder with different rudder angles. As can be recognized, the pressure difference between two sides of the rudder increases with the increase of rudder angle, which implies an increase of lift produced by the rudder, as shown in Figure 25. 


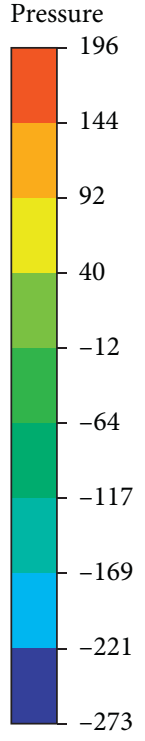

(Pa)

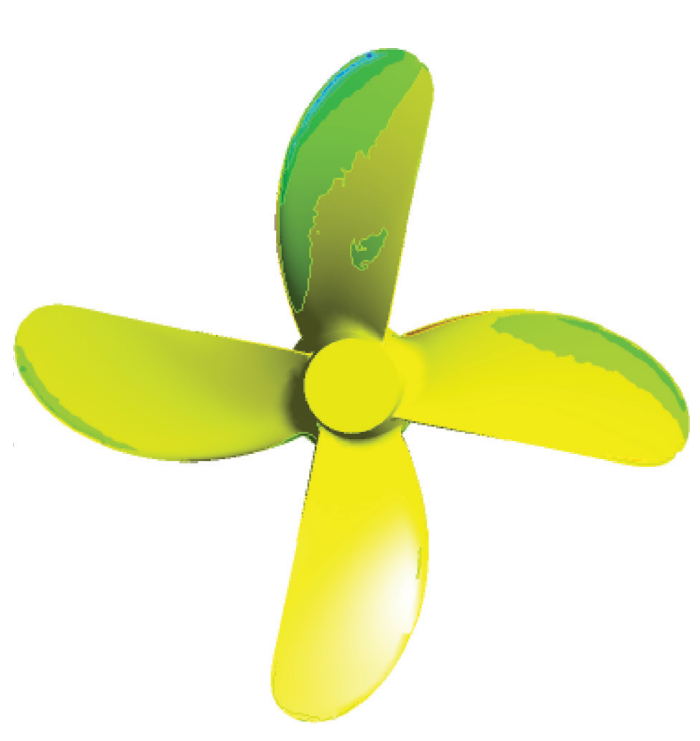

Front

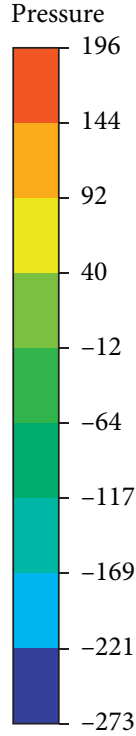

(Pa)

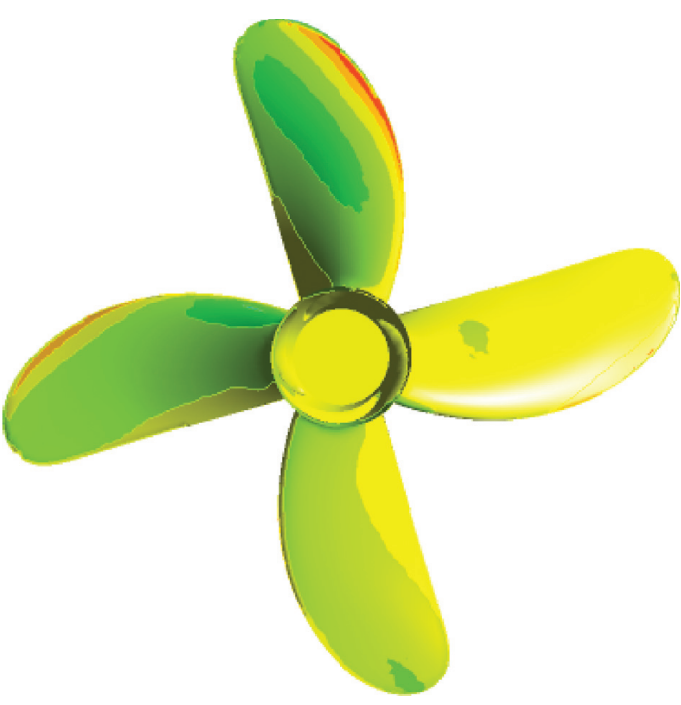

Back

FIGURE 20: Distribution of pressure on the propeller.

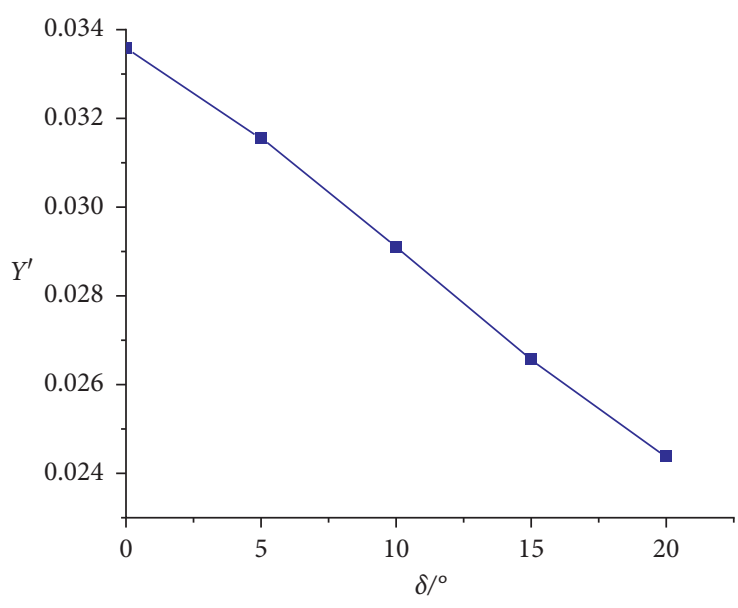

$-\theta=20^{\circ}$

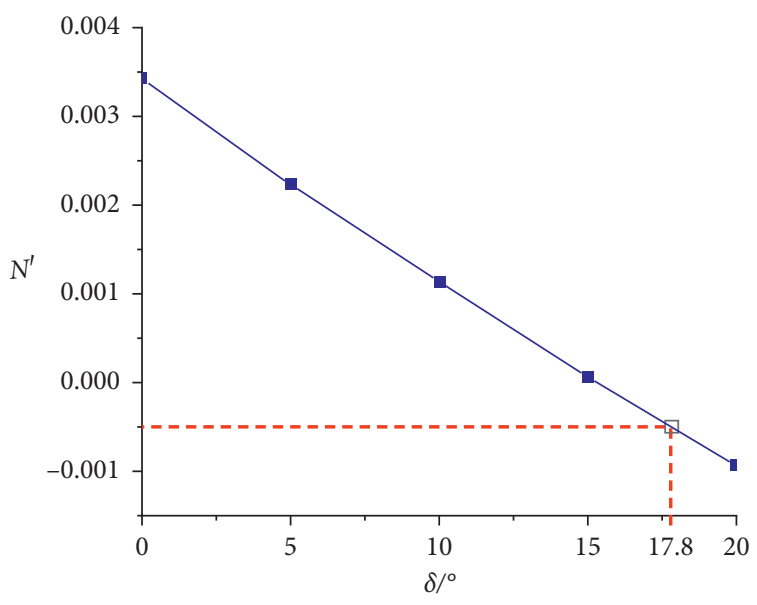

$-\theta=20^{\circ}$

(a)

(b)

Figure 21: Variation of transverse force and yaw moment with rudder angle.

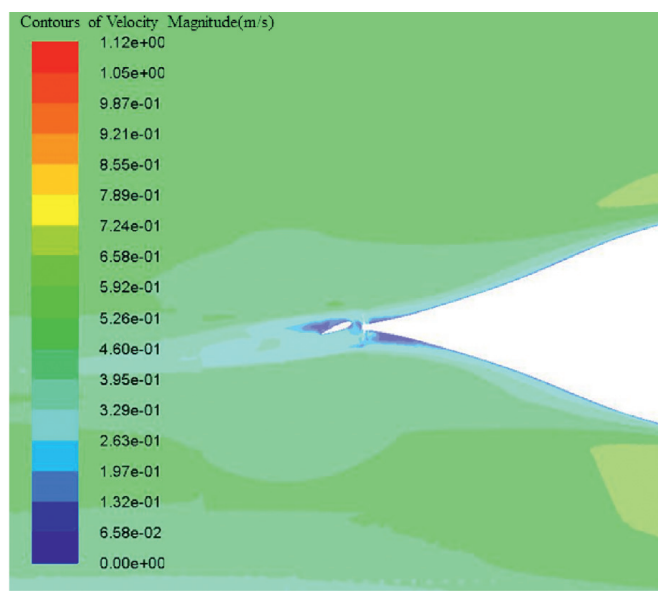

Figure 22: Velocity distribution at stern, around propeller, and rudder $\left(\delta=20^{\circ}\right)$. 


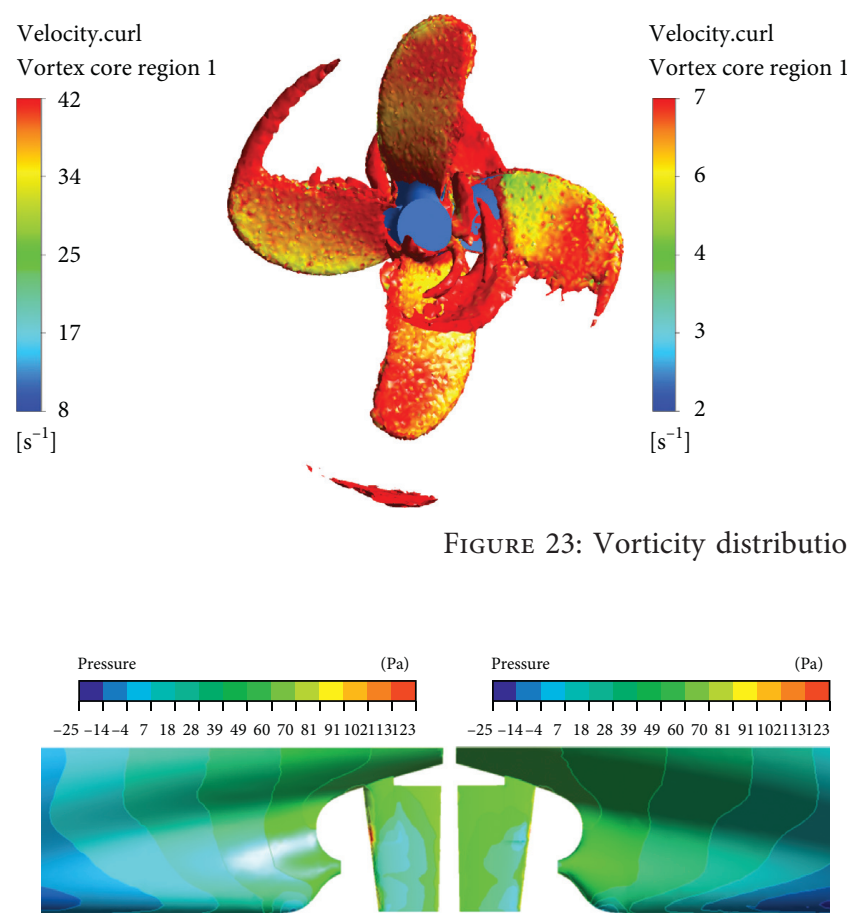

(a)
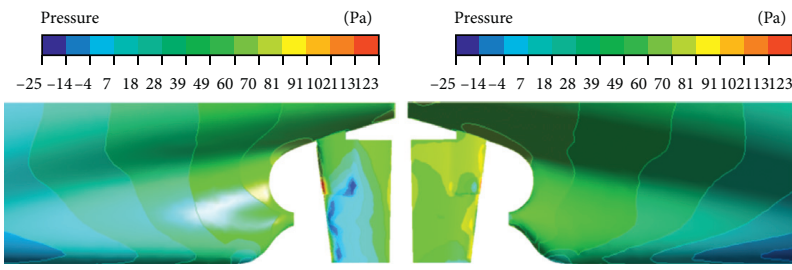

(c)
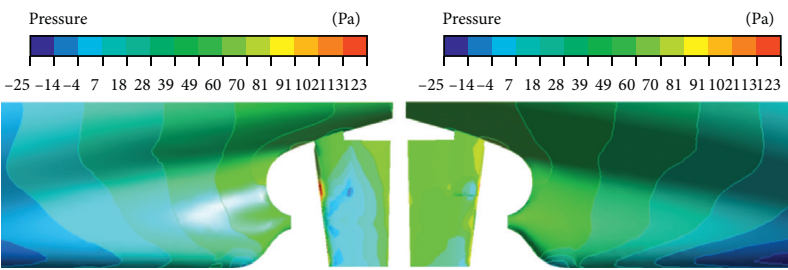

(b)
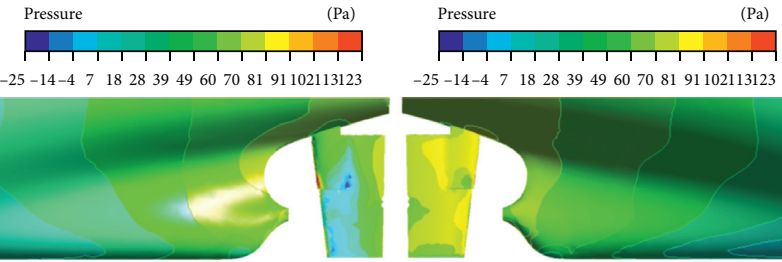

(d)

Figure 24: Variation of pressure on rudder surface with rudder angle. (a) $\delta=5^{\circ}$. (b) $\delta=10^{\circ}$. (c) $\delta=15^{\circ}$. (d) $\delta=20^{\circ}$.

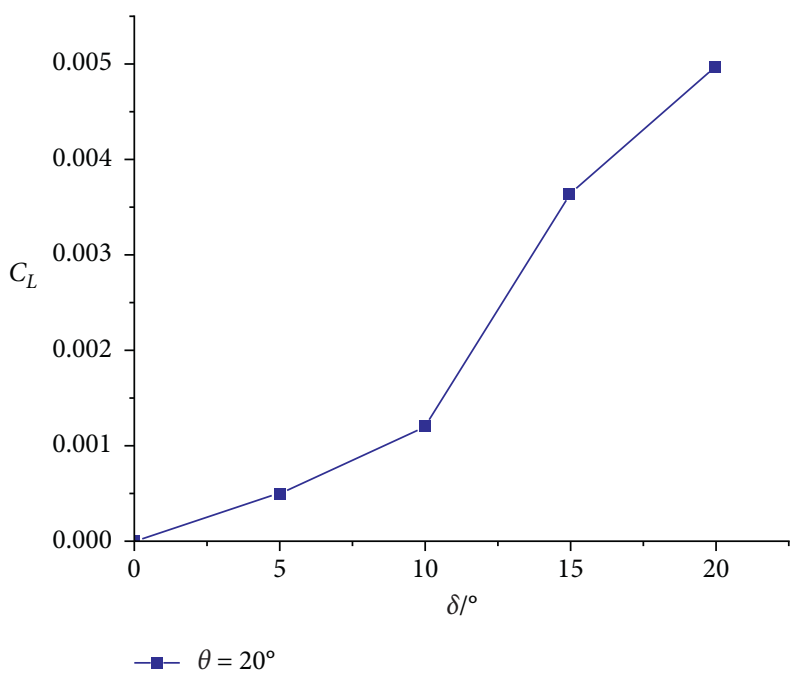

FIGURE 25: Variation of lift coefficient with rudder angle. 


\section{Conclusion}

In the study, the hydrodynamic force and moment of a KVLCC2 ship under the condition of near inclined banks are calculated by using CFD. Because of the small Froude number, the influence of wave making on free surface is ignored in the simulation. Combined with experiments, the verification and validation of the numerical calculation in the study are carried out, by which the calculation convergence and accuracy are guaranteed. Generally, the simulation results confirm the phenomenon of "bank effect." Three specific conclusions can be drawn from the analysis of simulation:

(1) For a ship sailing near a sloping bank, the transverse force and yaw moment increase with the decrease of ship-shore distance. At a fixed water depth, the greater the bank angle is, the greater the transverse force and yaw moment are.

(2) The direction of yaw moment of ships sailing near a sloping bank is more sensitive to drift angle than transverse force. Due to the bank suction, the direction of transverse force changes provided a large change of direction of drift angle while the direction of yaw moment changes for a small change of direction of drift angle.

(3) The yaw moment will vanish for a given rudder angle, which somehow alleviates the side effect of bank suction although for such a rudder angle the transverse force still exists. Such a special and specific rudder angle can be predicted by CFD calculation, as presented in the study.

In future work, the effects of free surface and ship speed will be taken into account. Moreover, different floating condition, e.g., rolling that sometimes happens to a ship in harbor, will be considered in manoeuvring simulation. In the study, the Multireference Frame model technology is used in the numerical simulation of hull-propeller-rudder model. As a steady-state approach, MRF has defects in dealing with unsteady problems. In future work, the nonuniform and unsteady characteristics of the flow field around the propeller will be considered.

\section{Data Availability}

Previously reported data were used to support this study and are available at DOI 10.1007/s00773-013-0221-6. The prior study (and datasets) is cited at relevant places within the text as reference [26].

\section{Conflicts of Interest}

The authors declare that they have no conflicts of interest regarding the publication of this paper.

\section{Acknowledgments}

The authors are thankful for the support by China Fujian Provincial Department of Ocean and Fisheries (MHGX-16).

\section{References}

[1] International Maritime Organization (IMO), "Standards for ship manoeuvrability," Resolution MSC, vol. 137, no. 76, 2002.

[2] J. Yao and Z. Zou, "Numerical study on bank effects for a ship traveling along banks," Journal of Wuhan University of Technology (Transportation Science and Engineering), vol. 3, pp. 5-8, 2011.

[3] The Maneuvering Committee of ITTC, "Final report and recommendations to the 25th ITTC," in Proceedings of the 25th International Towing Tank Conference, pp. 143-208, Fukuoka, Japan, September 2008.

[4] N. H. Norrbin, "Bank effect on a ship moving through a short dredged channel," in Proceedings of the 10th Symposium on Naval Hydrodynamcis, Cambridge, MA, USA, June 1976.

[5] E. Lataire, M. Vantorre, E. Laforce et al., "Navigation in confined waters: influence of bank characteristics on shipbank interaction," in Proceedings of the International Conference on Marine Research and Transportation, ICMRT, Naples, Italy, June 2007.

[6] A. Gronarz, "Investigation of the influence of a vertical wall on a ship moving with drift angle," in Proceeding of International Conference on Ship Manoeuvring in Shallow and Confined Water, Bank Effect, Antwerp Belguim, May 2009.

[7] D. C. Lo, D.-T. Su, and J.-M. Chen, "Application of computational fluid dynamics simulations to the analysis of bank effects in restricted waters," Journal of Navigation, vol. 62, no. 3, pp. 477-491, 2009.

[8] H. M. Wang, "Numerical study on the viscous flow and hydrodynamic forces on a manoeuvring ship in restricted waters," Doctoral Thesis, Shanghai Jiao Tong University, Shanghai, China, 2009.

[9] L. Zou, L. Larsson, G. Delefortrie et al., "CFD prediction and validation of ship-bank interaction in a canal," in Proceedings of the International Conference on Ship Manoeuvring in Shallow and Confined Water: Ship to Ship Interaction, Trondheim, Norway, May 2011.

[10] L. Zou and L. Larsson, "Computational fluid dynamics (CFD) prediction of bank effects including verification and validation," Journal of Marine Science and Technology, vol. 18, no. 3, pp. 310-323, 2013.

[11] K. Zhang and Z. J. Zou, "Numerical calculation of the hydrodynamic forces on a ship sailing along sloping bank," Journal of Hydrodynamics, vol. 29, no. 3, pp. 294-299, 2014.

[12] K. Zhang and Z. J. Zou, "Numerical calculation of hydrodynamic forces on ship sailing obliquely along sloping bank," Navigation of China, no. 3, pp. 52-56, 2015.

[13] H. W. Coleman and F. Stern, "Uncertainties and CFD code validation," Journal of Fluids Engineering, vol. 119, no. 4, pp. 795-803, 1997.

[14] ITTC, "QM. Procedure, Uncertainty analysis in CFD verification and validation methodology and procedures," vol. 7, 2002.

[15] C. D. Simonsen and F. Stern, "Verification and validation of RANS maneuvering simulation of Esso Osaka: effects of drift and rudder angle on forces and moments," Computers \& Fluids, vol. 32, no. 10, pp. 1325-1356, 2003.

[16] J. Kim, I. R. Park, S. H. Van et al., "Calculation of turbulent flows around a submarine for the prediction of hydrodynamic performance," Journal of Ship \& Ocean Technology, vol. 7, no. 4, pp. 16-31, 2003.

[17] Y. Tahra, R. V. Wilson, and P. M. Carrica, "RANS simulation of a container ship using a single-phase level-set method with overseer grid and the prognosis for extension to self- 
propulsion simulator," Marine Science Technology, vol. 11, pp. 209-228, 2006.

[18] N. Zhang, H. Shen, and H. Yao, "Uncertainty analysis in CFD for resistance and flow field," Journal of Ship Mechanics, vol. 12, no. 2, pp. 211-224, 2008.

[19] L. Zou, L. Larsson, and M. Orych, "Verification and validation of CFD predictions for a manoeuvring tanker," Journal of Hydrodynamics, vol. 22, no. 5, pp. 421-428, 2010.

[20] L. Zou and L. Larsson, "Computational fluid dynamics (CFD) prediction of bank effects including verification and validation," Journal of Marine Science and Technology, vol. 18, no. 3, pp. 310-323, 2013.

[21] C. Yang, R. Zhu, G. Miao, and J. Fan, "Uncertainty analysis in CFD for flow simulation around ship using RANS and DES," Journal of Shanghai Jiaotong University, vol. 46, no. 3, pp. 430-435, 2012.

[22] R.-c. Zhu, C.-1. Yang, G.-p. Miao, and J. Fan, "Computational fluid dynamics uncertainty analysis for simulations of roll motions for a 3D ship," Journal of Shanghai Jiaotong University (Science), vol. 20, no. 5, pp. 591-599, 2015.

[23] M. Simone, B. Ermina, A. H. Day et al., "Verification and validation of numerical modelling of DTMB 5415 roll decay," Ocean Engineering, vol. 162, pp. 209-223, 2018.

[24] H. Islam and C. G. Soares, "Uncertainty analysis in ship resistance prediction using OpenFOAM," Ocean Engineering, vol. 191, 2019.

[25] J. Hrvoje, V. Vuko, G. Inno et al., "CFD validation and grid sensitivity studies of full scale ship self propulsion," International Journal of Naval Architecture and Ocean Engineering, vol. 11, pp. 33-43, 2019.

[26] S. L. Toxopeus, C. D. Simonsen, E. Guilmineau, M. Visonneau, T. Xing, and F. Stern, "Investigation of water depth and basin wall effects on KVLCC2 in manoeuvring motion using viscous-flow calculations," Journal of Marine Science and Technology, vol. 18, no. 4, pp. 471-496, 2013.

[27] L. Eca, "On the influence of the iterative error in the numerical uncertainty of ship viscous flow calculations," in Proceedings of Symposium on Naval Hydrodynamics, Rome, Italy, September 2006.

[28] H. Liu, N. Ma, and X. C. Gu, "Manoeuvrability of a VLCC ship near a vertical bank and related course keeping control," in Proceedings of the International Conference on Ships and Offshore Structures, Hamburg, Germany, September 2016. 